\title{
Pulse-shape discrimination scintillators for homeland security applications
}

\author{
Mark E. Ellis*, Kirk Duroe and Paul A. Kendall \\ AWE Aldermaston, Reading, Berkshire RG7 4PR, United Kingdom \\ *mark.ellis@awe.co.uk
}

Published 1September 2016

\begin{abstract}
An extensive programme of research has been conducted for scintillation liquids and plastics capable of neutron-gamma discrimination for deployment in future passive and active Homeland Security systems to provide protection against radiological and nuclear threats. The more established detection materials such as EJ-301 and EJ-309 are compared with novel materials such as EJ-299-33 and p-terphenyl. This research also explores the benefits that can be gained from improvements in the analogue-to-digital sampling rate and sample bit resolution. Results are presented on the Pulse Shape Discrimination performance of various detector and data acquisition combinations and how optimum configurations from these studies have been developed into fieldready detector arrays. Early results from application-specific experimental configurations of multielement detector arrays are presented.
\end{abstract}

Keywords: Pulse shape discrimination; liquid scintillator; time correlation; differential die-away.

PACS numbers: 07.05.Hd, 29.40.Mc.

\section{Introduction}

The study into Pulse Shape Discrimination (PSD) performance in different detector geometries and configurations was born out of the desire to construct large area, costeffective fast neutron detectors. The primary purpose and key objective for this work is the application in passive and active Homeland Security systems.

PSD is a commonly used technique to distinguish between neutron and gamma ray events occurring in the same detector volume that might not otherwise be possible from measuring energy deposition alone. There are several scintillation materials available that have the property of yielding light pulses of different shape for different interacting particle types. Examples of such materials are EJ-301 and EJ-309, in which neutrons produce a light pulse that has a longer tail than gamma rays due to the way the particles give up their energy in the liquid. ${ }^{1}$

This is an Open Access article published by World Scientific Publishing Company. It is distributed under the terms of the Creative Commons Attribution 3.0 (CC-BY) License. Further distribution of this work is permitted, provided the original work is properly cited. 


\section{Pulse-shape Discriminating (PSD) Scintillation Detectors}

There are a wide range of radiation detectors that fall under the category of PSD scintillators. This work focuses on liquid scintillators such as Eljen EJ-309 and EJ-301, plastic scintillators such as EJ-299-33, and crystalline scintillators such as p-terphenyl. Over the past four years, AWE has had a specific programme of work investigating the performance of these types of PSD scintillators and in particular how they may be applied to passive detection (e.g. radiation portal at a port or choke point) and active detection (typically targeted to primary and secondary inspection at a port).

\subsection{PSD technique and processing}

The charge pulse from the detector photomultiplier tube (PMT) anode output is captured by a digitizer that converts the analogue charge pulse to a digital representation, which is subsequently processed for energy and PSD. This is shown in Fig. 1.

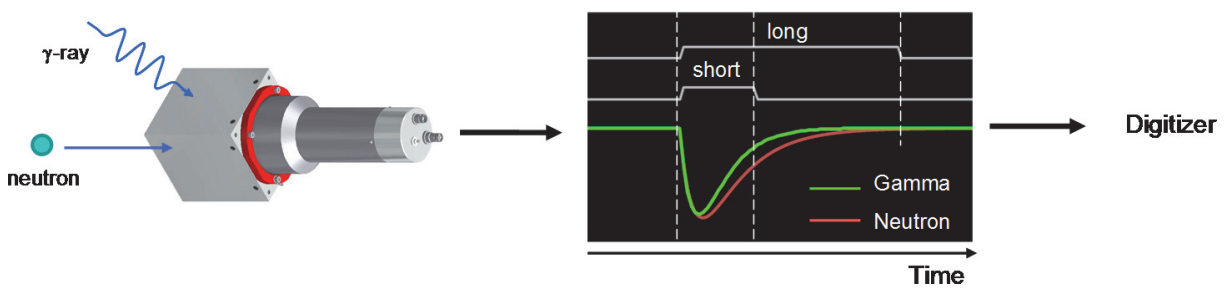

Fig. 1. PSD processing chain showing the charge pulse shape difference for neutrons and gamma rays.

The CAEN series of digitizers used in these experiments are capable of performing on-board real-time PSD but also with an option of applying a different PSD algorithm off-line. All results presented here are for the real-time on-board processing algorithm.

The CAEN PSD algorithm works on the charge integration technique, ${ }^{2}$ where the relative amounts of charge from the prompt and delayed fluorescence from the scintillation medium is used to separate neutron and gamma-ray interactions. ${ }^{3}$ To achieve this, the detector charge pulse is integrated over a short time period (short gate) and also a longer time period (long gate). The relative amount of charge integrated over these two gates determines whether the interaction was from a neutron or gamma ray.

For every detected radiation event, the digitizer outputs a record in the list-mode output file containing the time stamp, short gate charge, long gate charge, and a PSD parameter calculated according to the following formula:

$$
P S D=\left(Q_{\text {long }}-Q_{\text {short }}\right) / Q_{\text {long }},
$$

where $Q_{\text {short }}$ and $Q_{\text {long }}$ are the charge integrated during the short time-gate and long timegate, respectively. The pulse-processing and PSD technique described above has been used for all of the experimental investigations presented in this paper. 


\subsection{Figure of Merit (FOM)}

The output list-mode data file from a measurement (as described in Sec. 2.1) can be displayed on a contour plot of energy (keVee) versus a PSD value as defined in Eq. (1). The energy and PSD values are 'binned' as appropriate and the number of events (counts) in each energy and PSD 'bin' is represented as color intensity. An example contour plot for a ${ }^{252} \mathrm{Cf}$ measurement with the $76 \times 76 \mathrm{~mm}$ EJ-309 cylinder detector is shown in Fig. 2.
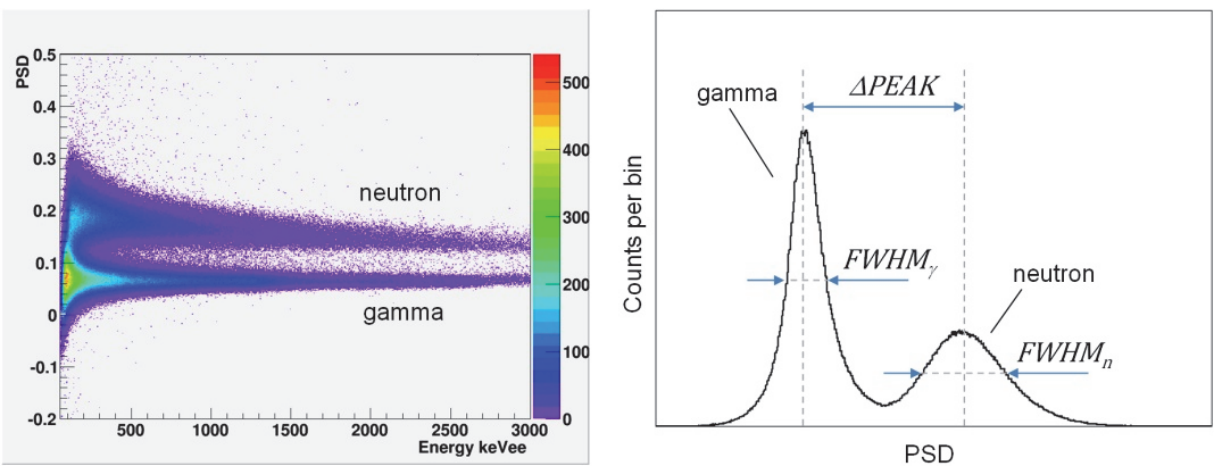

Fig. 2. Contour PSD plot for a ${ }^{252} \mathrm{Cf}$ measurement with a 76 x $76 \mathrm{~mm}$ cylinder EJ-309 detector (left) and an example plot of counts per bin versus PSD parameter over a given energy range (right).

A Figure of Merit (FOM) is defined as a measure of separation between gamma and neutron distributions. The right hand plot of Fig. 2 represents a projection of the contour plot onto the PSD axis over a given energy range.

The FOM used here is proportional to the separation of the gamma and neutron peaks $(\triangle P E A K)$ and inversely proportional to their spread $\left(F W H M_{\gamma}\right.$ and $F W H M_{n}$ for the gamma-ray and neutron peaks respectively), defined as:

$$
F O M=\triangle P E A K /\left(F_{W H M_{\gamma}}+F W H M_{n}\right) .
$$

There are other definitions that could be used as the FOM, but this is the one most commonly used by others. ${ }^{4}$

\subsection{PSD performance vs. volume/shape}

It is known that there is a variation in light pulse shapes from neutrons and gamma rays as the shape and volume of the detection medium is varied. ${ }^{5}$ A study was carried out to investigate this variation and to decouple this effect from other complicating effects such as the data acquisition electronics (and how it is configured) and PSD algorithms.

Initial laboratory measurements were carried out with Eljen EJ-309 liquid scintillators in different geometries with a ${ }^{252} \mathrm{Cf}$ source. The full details of the study can be found in Ref. 5, but the main findings that are relevant to this paper are summarized here. The PSD performance was assessed for detector volumes varying from 350 cubic centimeters to 6570 cubic centimeters in cubed and right-cylindrical geometries. The key finding from this study was that there is a steady degradation of PSD performance with 
increasing detection volume, but the degradation is worse at higher energies. Importantly, the extent of this performance degradation has been quantified. Also found is that cube geometries perform just as well as cylinders (while presenting an $8 \%$ greater surface area for the same detection volume). The FOM performance comparisons for volume and shape are shown in Fig. 3.
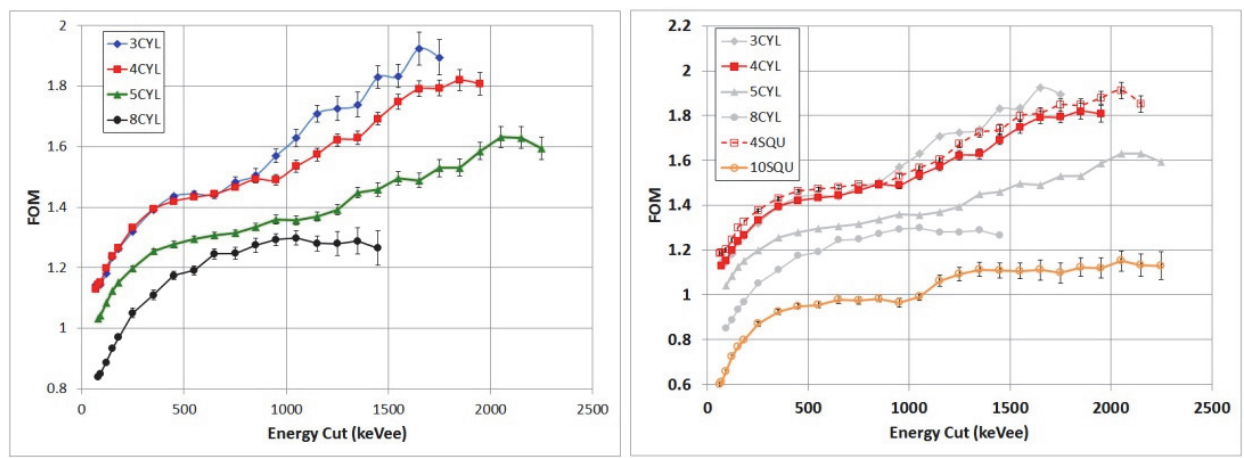

Fig. 3. FOM calculations for ${ }^{252} \mathrm{Cf}$ source at different energy cuts for EJ-309 cylinders ranging from 3 inches (3CYL) up to 8 inches (left) and a 4 inch cube (4SQU) compared with a 4 inch cylinder (right).

\subsection{PSD material \& Data Acquisition (DAQ) comparisons}

The study described in Sec. 2.3 focused on PSD performance in liquid scintillators and in particular, the EJ-309 high flashpoint material. But there are other materials, some of which out-perform EJ-309 but are not desirable for large-area detector arrays due to either cost or being a hazardous material. However, it is interesting and necessary to compare EJ-309 with other available detector technologies that exhibit PSD properties.

A collaborative experimental workshop (with the Naval Research Laboratory in Washington, DC) was held to investigate how PSD performance is affected by detector volume, shape, and detection material as well as the particular DAQ system used. Measurements were made with a total of eight different detector types and seven different DAQ systems. The full details of the study can be found in Ref. 6, but the main findings that are relevant to this paper are summarized here.

\subsubsection{Detection material}

These experiments showed consistently that the original EJ-301 material is still the benchmark material for PSD performance. The only other material that was shown to match the performance of EJ-301 is p-terphenyl, which shows comparable PSD FOM figures. P-terphenyl is a solid material rather than a liquid and does not present the same toxic and flammable hazards as EJ-301, but there are limitations in the crystal growth size (76 mm being the largest that could be easily procured to date), and it remains a relatively expensive material (maybe 25\% more than EJ-309, based on a 76 x $76 \mathrm{~mm}$ detector). 
For the reasons given above, the designs that have been developed for the specific application described later in Secs. 3 and 4 use EJ-309 scintillators, but the study shows that EJ-299-33 ${ }^{7-8}$ would be a viable alternative since the PSD performance is only slightly worse than EJ-309 but it has the benefit of being a solid rather than a liquid, which is highly desirable in an operational scenario.

\subsubsection{Data acquisition electronics}

The experiments carried out at this workshop show how the PSD performance (determined as described in Sec. 2.2) is affected to a large extent by the data acquisition electronics used, whose specification may vary in parameters such as sampling rate and ADC resolution but also by other factors such as the pulse-processing algorithms employed. ${ }^{9,10}$ These experiments showed up to a four-fold variation in FOM for any given detector depending on the data acquisition system used. ${ }^{6}$ The results from this workshop have been used to inform the choice of data acquisition digitizer used on the designs developed for the applications described later.

\section{Passive Detection}

AWE has an on-going research activity into passive detection of Special Nuclear Material (SNM) for Homeland Security applications. As part of this research, AWE has developed a full scale prototype demonstrator system, which has just completed its first phase of trials. In addition to this, AWE is investigating the use of PSD scintillators as an alternative to the detector technology currently employed in the demonstrator system.

\subsection{Prototype demonstrator}

Following a wide range of investigations into passive radiation techniques and detector performance assessments, several technologies were selected for the prototype. The system comprises two subsystems, one based on high resolution gamma spectroscopy using High Purity Germanium (HPGe) and the other based on a time-correlation technique developed by Arktis Radiation Detectors Ltd utilizing polyvinyl toluene (PVT) and ${ }^{4} \mathrm{He}$ detectors. The time-correlation technique was developed under a collaboration known as the "FLASH Portals Project", ${ }^{11}$ hereafter referred to as FLASH.

\subsubsection{FLASH Portals Project}

The FLASH system comprises two portal panels, each containing two large PVT detectors and eight ${ }^{4} \mathrm{He}$ detectors developed by Arktis. The ${ }^{4} \mathrm{He}$ detectors are sensitive to fast neutrons and gamma rays and utilize Pulse-shape Discrimination (PSD) to distinguish between the particle types. ${ }^{11}$ In addition to gamma-ray and neutron gross counting techniques typical of Radiation Portal Monitors (RPMs), the FLASH system was designed to use the cross-correlation signatures, outlined by A. Enqvist et al., ${ }^{12}$ to distinguish SNM from other sources of radiation. 
Several signatures were investigated as part of the FLASH project; one such signature used the cross-correlation between PVT detectors in opposite sides of the portal. Typical signatures for ${ }^{252} \mathrm{Cf}$, background and $\mathrm{KCl}(\mathrm{NORM})$ are given in Fig. 4.
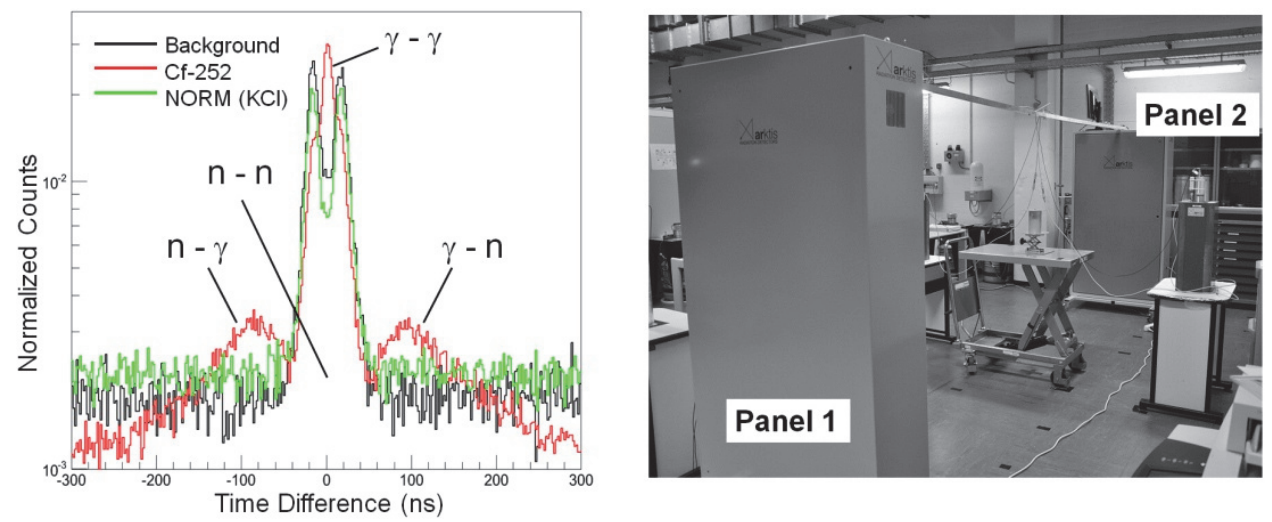

Fig. 4. Cross-correlation measurements between PVT detectors in opposite sides of the portal. The time difference is equal to the time of an event in panel 1 minus the time of the correlated event in panel 2; panel numbering is arbitrary. The number of events has been normalized by the total number of events.

Features in the ${ }^{252} \mathrm{Cf}$ data include a peak at $\mathrm{t}=0$ corresponding to correlated $\gamma-\gamma$ and $\mathrm{n}-\mathrm{n}$ events and peaks at $\mathrm{t}= \pm 100 \mathrm{~ns}$ corresponding to $\mathrm{n}-\gamma$ or $\gamma-\mathrm{n}$ events. These features do not appear in the background and NORM data due to the lack of correlated events; peaks at $\mathrm{t}= \pm 15 \mathrm{~ns}$ are consistent with gamma events scattered between the two sides of the portal.

\subsection{PSD scintillators for passive radiation detection applications}

Our applications typically involve scanning cargo containers or vehicles to ensure they are clear of radioactive materials. This requires sensitivity over many square meters and a stand-off of several meters. Vehicles may be moving, providing a dynamic field and limited counting times. In addition to these practical considerations, there is the desire to detect the smallest true positive signals possible. All of which point to a requirement for large detectors, or at least a large detector coverage. It has also been shown in Sec. 2.3 that PSD performance is a function of detector geometry, limiting the useful sizes.

It was found that $102 \times 102 \times 102 \mathrm{~mm}$ EJ-309 cube detectors provided a good tradeoff between PSD performance and cost per surface area. A large area array of such detectors, consisting of 49 EJ-309 liquid scintillators in a 7 x 7 arrangement, has been constructed to provide a configurable test bed for fast neutron detection techniques on the scale that would be necessary for passive detection applications; this is shown in Fig. 5.

Moving to greater numbers of detectors introduces further complexity to the choice of Data Acquisition (DAQ) system used. Relatively recent developments in digital data acquisition systems provides the capability for high channel-density solutions, with several solutions offering digital signal processing capable of performing on-board PSD 
(implemented on a programmable hardware device) and list-mode read out. The detector array shown in Fig. 5 utilizes seven CAEN V1751 digitizer cards (1 GSamples/s and 10 bit ADC resolution) that perform on-board PSD based on the charge integration method. ${ }^{2}$ This array can be used as a single $1 \mathrm{~m}^{2}$ panel or reconfigured to explore a variety of passive detection techniques.

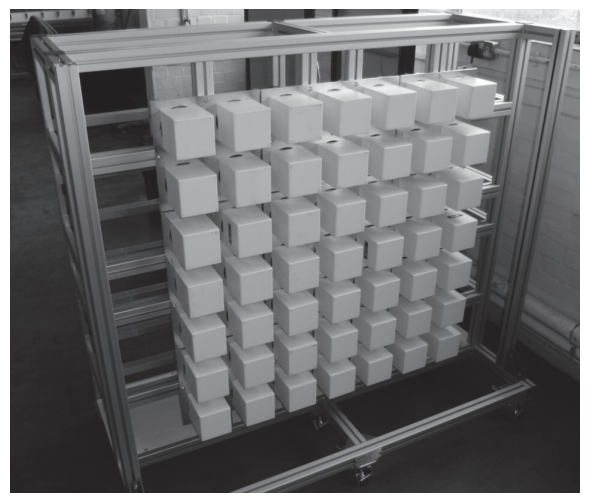

Fig. $5.7 \times 7$ array of $102 \times 102 \times 102 \mathrm{~mm}$ cube EJ-309 liquid scintillator detectors used as a test bed for applying liquid scintillator detectors to different applications.

\subsection{Time correlation with liquid scintillators}

The detector array shown in Fig. 5 was reconfigured into two arrays separated by $2 \mathrm{~m}$, each containing 16 detectors in a 4 x 4 arrangement. The aim was to mimic a two-panel radiation portal and to reproduce the results from the FLASH experiments. ${ }^{11}$ Using the PSD capability of liquid scintillators, particle types can be identified and different signatures distinguished, as previously demonstrated by A. Enqvist et al. ${ }^{12}$ This provides an opportunity to develop techniques such as those demonstrated with the FLASH system and extend them through the separate treatment of particle signatures and potentially improve the discrimination capability of a passive portal system. Figure 6 shows the separated signatures, which can be compared to the FLASH results (see Fig. 4).
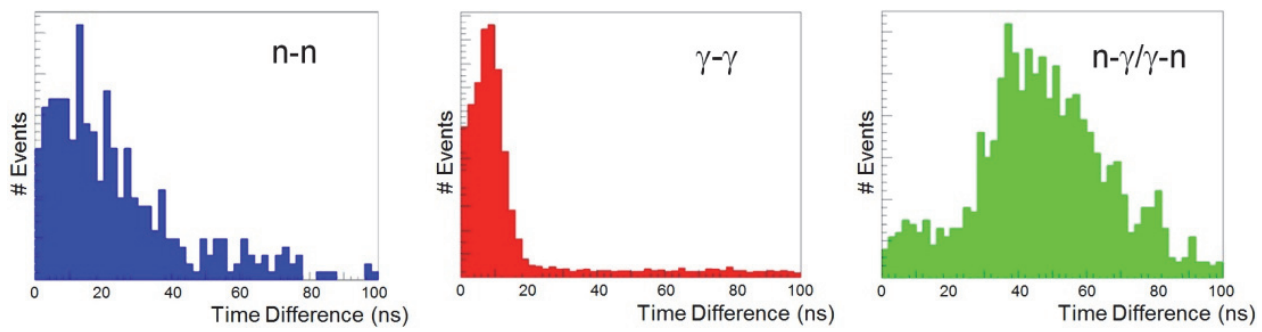

Fig. 6. Cross-correlation measurements between the two panels of liquid scintillators. The plots compare very well with the original FLASH results; the one sided nature of the graph is due to time differences being calculated between events in sequence rather than between fixed detector numbers. Note: The only available ${ }^{252} \mathrm{Cf}$ source was small $(16 \mathrm{kBq})$ and inside a lead pot, which is why the $\gamma-\gamma$ signature appears more like background (dominated by scattering). 


\section{Active Detection}

AWE has an ongoing research activity into active detection of Special Nuclear Material (SNM) for Homeland Security applications. As part of this research, AWE is collaborating with the US Domestic Nuclear Detection Office (DNDO) and Rapiscan Systems Inc. to develop a full scale prototype demonstrator system, which is currently undergoing its first phase of trials. In addition to this, AWE is investigating the use of PSD scintillators in an active interrogation environment.

\subsection{Differential die-away with liquid scintillators}

One area where it was felt that PSD scintillators could be beneficial for an active interrogation system is with the Differential Die-Away (DDA) technique. This technique was first developed by Los Alamos National Laboratory (LANL) in the early 1980s for the quantitative assay of generic transuranic waste from nuclear reactors. ${ }^{13}$

The DDA technique is based on measurements of the decay time constants of neutron flux in a diffusing medium (i.e. the object under inspection). Thermal neutrons will decay exponentially due to absorption and leakage with a time constant typically in the region of hundreds of microseconds up to a few milliseconds (depending on the object content). ${ }^{14}$ Fast neutrons will decay exponentially due to leakage and thermalization with a time constant several orders of magnitude faster than the thermal time constant.

If fissile material is present within the object under inspection, then thermal neutrons will create fast neutrons though the fission process. Fast neutrons created in this way will decay at the same rate as the thermal neutrons that are creating them.

\subsubsection{Detectors for the DDA technique}

The differential die-away technique requires a detector that is sensitive only to fast neutrons. If no fissile material is present in the object under inspection, then the detector signal (to fast neutrons) will decay at the characteristic decay constant of the detector (determined by the neutron life-time within the detector before detection). If fissile material is present, then the detector signal will decay at the thermal time constant rate, which is itself dependent upon the material contained within the object. ${ }^{15}$

The commonly used detector for DDA is the ${ }^{3} \mathrm{He}$ proportional counter with a moderator 'build-up' that makes it predominantly sensitive to fast neutrons. ${ }^{14}$ A typical ${ }^{3} \mathrm{He}$ detector will have a characteristic decay constant (die-away time) of tens of microseconds. An AWE internal study identified liquid scintillators as a viable alternative to ${ }^{3} \mathrm{He}$ since they should exhibit superior die-away characteristics (nanoseconds rather than microseconds) and should allow the DDA signature to be accessed earlier in time.

\subsubsection{Initial DDA measurements with liquid scintillators}

An eight-element detector array was designed for the purpose of measuring the characteristic decay constant (die-away time) of EJ-309 liquid scintillators. The detectors 
were constructed in a $2 \times 4$ arrangement of $102 \times 102 \times 102 \mathrm{~mm}$ cube detector cells with provision for steel/lead shielding, as shown in Fig. 7.

Measurements were made with a DT (deuterium-tritium) generator. Early results of these die-away measurements can be seen in Fig. 7, which shows the die-away obtained from the liquid scintillators with and without the presence of fissile material (Low Enriched Uranium - LEU) and compares this with a boron Proportional counter (PC), which has similar die-away characteristics as a ${ }^{3} \mathrm{He}$ detector. The liquid scintillator dieaway, which is almost instantaneous compared to that of the boron proportional counter, means that the integration of the DDA signature can be started at an earlier time.
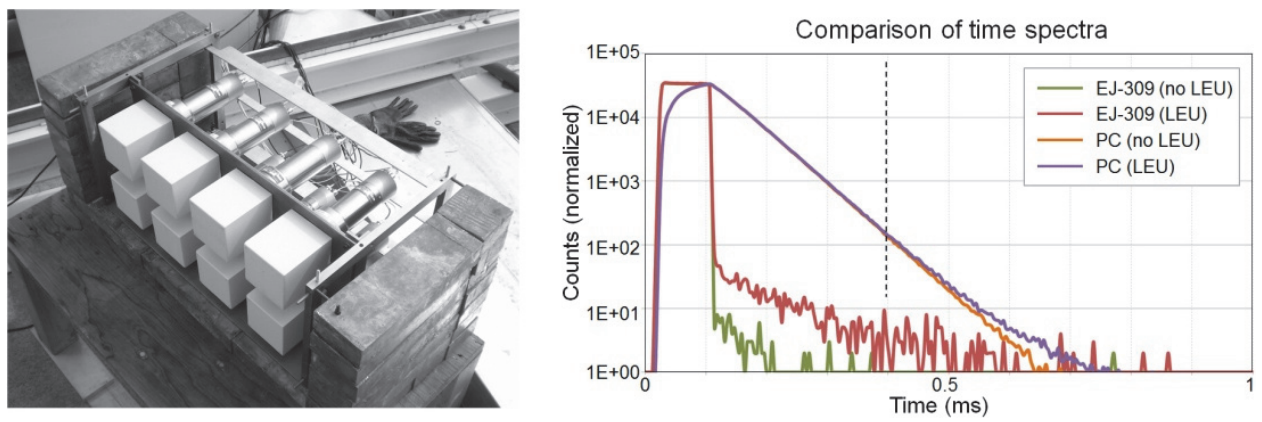

Fig. 7. 2 x 4 array of $102 \times 102 \times 102$ mm EJ-309 liquid scintillator detectors used for characteristic die-away measurements. Die-away time spectra for EJ-309 liquid scintillators and a boron proportional counter (PC).

In addition to the fast die-away time, liquid scintillators bring other advantages such as removing the need for a bulky polyethylene moderator (although some high-Z shielding may be needed) and the inherent detection of gamma rays, which could be used as an additional signature in an active detection system. However, it is appreciated that liquid scintillators (and PSD scintillators in general) are difficult to operate in an active environment where the gamma flux can be particularly high and performing PSD becomes difficult.

To explore this further, PSD measurements have been made for a ${ }^{252} \mathrm{Cf}$ source with increasing gamma ray fields. Figure 8 shows how the separation of neutron and gamma events deteriorates as the gamma field increases from $0.6 \mathrm{mR} / \mathrm{h}$ to $20 \mathrm{mR} / \mathrm{h}$. The current work is to investigate how the (wanted) neutron signal is being corrupted and importantly how it can be recovered by sophisticated pulse processing. 

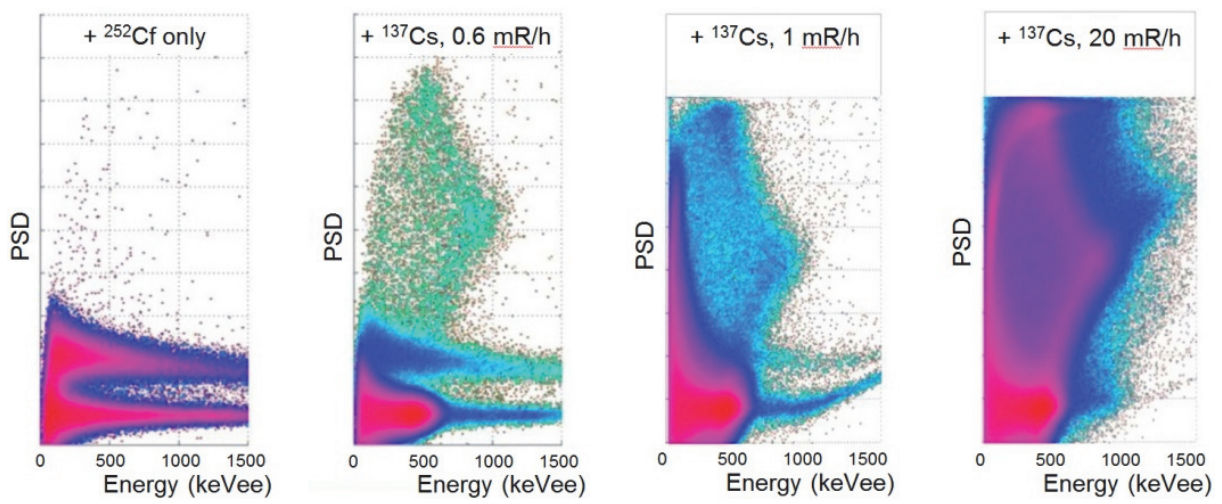

Fig. 8. Illustration of the degradation in neutron-gamma separation from a ${ }^{252} \mathrm{Cf}$ source as the gamma field $\left({ }^{137} \mathrm{Cs}\right)$ is increased from $0.6 \mathrm{mR} / \mathrm{h}$ to $20 \mathrm{mR} / \mathrm{h}$.

\section{Summary}

A wide range of fundamental research into PSD scintillator performance has been conducted as well as a significant body of research on PSD performance with different data acquisition systems. It has been demonstrated how small-scale systems have been designed and built based on the earlier performance studies and applied to both passive and active Homeland Security applications (correlated particles and differential dieaway). The benefits of liquid scintillators for these applications have been demonstrated, and the challenges, particularly in an active environment, have been highlighted.

The work presented here is the first stage of an ongoing programme of research at AWE looking at the detection of Radiological and Special Nuclear Material. For the past few years the focus has been on border security, but looking forward, the application areas will be expanded to include wide area search, counter terrorism, arms control verification, and others. Future research will look at using PSD scintillators in these application areas, but it is likely that there will be a gradual move from liquids to solid plastics as materials such as EJ-299-33 become more prevalent.

\section{Acknowledgments}

The authors thank UK Home Office and Ministry of Defence for funding this research.

\section{References}

1. T. K. Alexander and F. S. Goulding, Nucl. Instr. Meth. A 13, 244 (1961).

2. G. Ranucci, Nucl. Instrum. Meth. A 354, 389 (1995).

3. G. F. Knoll, Radiation Detection and Measurement, 4th edn. (Wiley, 2010).

4. J. R. M. Annand, Nucl. Instr. Meth. A 262, 337 (1987).

5. M. Ellis, C. Tintori, P. Schotanus, K. Duroe, P. A. Kendall and G. Mini, The Effect of Detector Geometry on EJ-309 Pulse Shape Discrimination Performance, in IEEE Nuclear Science Symposium Conference Record, N20-1, 2013. 
6. P. A. Kendall, K. Duroe, P. R. Arthur, M. Ellis, M. C. Owen, R. S. Woolf, E. A. Wulf, A. L. Hutcheson and B. Phlips, Comparative Study of the Pulse Shape Discrimination (PSD) Performance of Fast Neutron Detectors, in IEEE Nuclear Science Symposium Conference Record, N25-5, 2014.

7. S. A. Pozzi, M. M. Bourne and S. D. Clarke, Nucl. Instr. Meth. A 723, 19 (2013).

8. D. Cester, G. Nebbia, L. Stevanato et al., Nucl. Instr. Meth. A 735, 202 (2014).

9. M. Flaska, M. Faisal et al., Nucl. Instr. Meth. A 729, 456 (2013).

10. D. Cester, M. Lunardon, G. Nebbia et al., Nucl. Instr. Meth. A 748, 33 (2014).

11. D. Murer, D. Blackie and P. Peerani, Benefits of Time Correlation Measurements for Passive Screening, in Applications of Nuclear Techniques (CRETE13), International Journal of Modern Physics: Conference Series 27, 1460132 (2014).

12. A. Enqvist, M. Flaska and S. Pozzi, Nucl. Instr. Meth. A 595, 426 (2008).

13. J. T. Caldwell, R. D. Hastings, G. C Herrera, W. E. Kunz et al., The Los Alamos SecondGeneration System for Passive and Active Neutron Assays of Drum-Size Containers, Los Alamos National Laboratory LA-10774-MS (1986).

14. K. A. Jordan and T. Gozani, Nucl. Instr. Meth. B 261, 365 (2007).

15. T. J. Shaw, D. A. Strellis, J. Stevenson, D, Keely and T. Gozani, Fissile Material Detection by Differential Die Away Analysis, in AIP IP Conf. Proc. 1099, 633 (2009). 